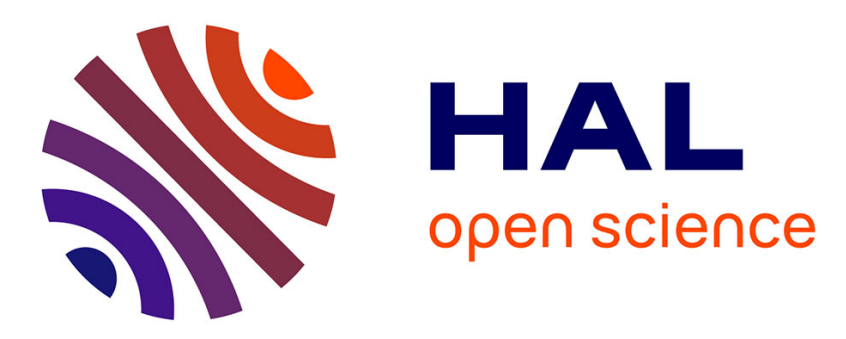

\title{
Light and thermally induced metastabilities in electrochemically etched nanocrystalline porous silicon
}

N P Mandal, Manohar Awasthi, Aniruddha Konar, Abhishek Kumar, Dudhnath Patel

\section{- To cite this version:}

N P Mandal, Manohar Awasthi, Aniruddha Konar, Abhishek Kumar, Dudhnath Patel. Light and thermally induced metastabilities in electrochemically etched nanocrystalline porous silicon. Philosophical Magazine, 2009, 89 (04), pp.311-321. 10.1080/14786430802620716 . hal-00514005

\section{HAL Id: hal-00514005 \\ https://hal.science/hal-00514005}

Submitted on 1 Sep 2010

HAL is a multi-disciplinary open access archive for the deposit and dissemination of scientific research documents, whether they are published or not. The documents may come from teaching and research institutions in France or abroad, or from public or private research centers.
L'archive ouverte pluridisciplinaire HAL, est destinée au dépôt et à la diffusion de documents scientifiques de niveau recherche, publiés ou non, émanant des établissements d'enseignement et de recherche français ou étrangers, des laboratoires publics ou privés. 


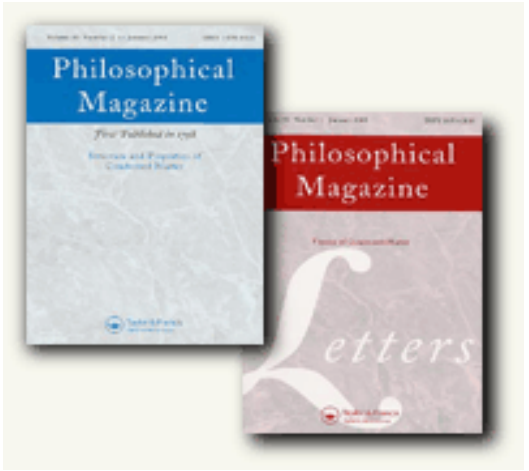

\section{Light and thermally induced metastabilities in electrochemically etched nanocrystalline porous silicon}

\begin{tabular}{|r|l|}
\hline Journal: & Philosophical Magazine \& Philosophical Magazine Letters \\
\hline Manuscript ID: & TPHM-08-Jul-0241.R1 \\
\hline Journal Selection: & Philosophical Magazine \\
\hline Author: & 30 -Aug-2008 \\
\hline Complete List of Authors: & $\begin{array}{l}\text { Mandal, N; UCL, EE } \\
\text { Awasthi, Manohar; Humboldt-Universität zu Berlin,, Physics } \\
\text { Konar, Aniruddha; University of Notre dame,, Physics } \\
\text { Kumar, Abhishek; , University of Massachusetts Lowell, Physics } \\
\text { Patel, Dudhnath; IIT Kanpur, physics }\end{array}$ \\
\hline Keywords: & $\begin{array}{l}\text { a-Si:H, electrical conductivity, electrochemistry, electron } \\
\text { paramagnetic resonance, nanostructured semiconductors, } \\
\text { photoluminescence }\end{array}$ \\
\hline Keywords (user supplied): & a-Si:H, electrical conductivity, electrochemistry \\
\hline
\end{tabular}

\section{5 scholarONE \\ Manuscript Central}




\title{
Light and thermally induced metastabilities in electrochemically etched nanocrystalline porous silicon
}

\author{
N. P. Mandal, ${ }^{1}$ Manohar Awasthi, ${ }^{2}$ Aniruddha Konar, ${ }^{3}$ Abhishek Kumar ${ }^{4}$ and D. N. \\ Patel $^{1}$ \\ ${ }^{1}$ Department of Physics, Indian Institute of Technology, Kanpur, 208016, India. \\ ${ }^{2}$ Humboldt-Universität zu Berlin, Institut für Physik, AG Moderne Optik, \\ Hausvogteiplatz 5-7, D-10117 Berlin, Germany \\ ${ }^{3}$ Department of Physics and Department of Electrical Engineering, University of \\ Notre dame, Notre Dame, Indiana 46556, USA. \\ ${ }^{4}$ Department of Physics and Applied Physics, University of Massachusetts Lowell \\ One University Avenue, Lowell, Massachusetts 01854
}

\begin{abstract}
We observe that light soaking for short durations and thermal quenching in nanocrystalline porous silicon (PS) produce metastable states. These metastable states show higher dark current and photo current, large photoluminescence and weaker electron spin resonance (ESR) signal. However, long exposures to light produce opposite effect. The metastable states are stable against sub band gap light exposures. These metastable states can be removed by annealing at $150{ }^{\circ} \mathrm{C}$ for 1 hour. ESR shows the presence of a-Si phase $(\mathrm{g} \sim 2.0058,6.4 \mathrm{G})$ in PS sample, but it is not sufficient to explain all experimental results. Rather, our experiments suggest that light soaking causes more than one type of defects in porous silicon. The structural changes involving the movement of hydrogen present at the surface of PS or at PS/aSi interface may be responsible for these effects.
\end{abstract}




\section{INTRODUCTION}

Porous silicon (PS) consists of nanometer size crystallites of silicon and shows photoluminescence (PL) in the visible region at room temperature. It has attracted attention of many researchers in recent years because of easy processing at room temperature. PS also offers myriad of opportunities for various applications in optoelectronic devices, bio-sensors [1], and gas sensors [2, 3, 4, 5]. The PL spectrum is broad, and often asymmetric about the PL peak energy with an exponential tail on low energy side. However, PL from PS is sensitive to various treatments, e.g., exposure to light or ambient gases $[1,5]$. These give rise to metastabilities which persist for a long time even at room temperature. For example, short exposures to light produce a state whose dark current (DC) is higher than annealed state DC (persistent photoconductivity, PPC) and persists for several days at room temperature $[6,7,8]$. Xu et al and Tischler et al. $[9,10]$ have found that PL does not change when light soaking is done in vacuum or nitrogen atmosphere respectively. On the other hand, Matsumoto and co workers [11] reported that long exposures of light to PS in vacuum caused a decrease in PL and increase in dangling bond density (ESR signal).

We have observed the effect of light soaking (LS) and fast thermal quenching (FQ) on PS to see the role of a-Si:H, if it is at all present. We observe that short exposures of visible light to PS in vacuum leads to increase in PL intensity. The photocurrent (PC) and DC are found to increase, while ESR decreases. On the other hand, changes are in opposite direction for long exposures and are similar to well known Staebler-Wronski (SW) effect in hydrogenated amorphous silicon [12]. Fast thermal quenching (FQ) from $450 \mathrm{~K}$ to room temperature also gives an increase in $\mathrm{DC}$, PC and PL and a decrease in ESR signal. Though the increase in DC after fast cooling 
and short exposures in case of PS is similar to PPC that was observed in doped a$\mathrm{Si}: \mathrm{H}$, no decrease in ESR has so far been reported for undoped a-Si:H $[13,14,15]$. Furthermore, LS was performed at three different temperatures to prove that LS causes more than one type of defects in porous silicon. Since, ESR measurement show the presence of a-Si phase in PS one might argue that all metastabilities in PS may be caused by a-Si. A closer look, however, suggests this may not be so. For example, we find that the combined effect of $\mathrm{NH}_{3}$ vapor exposure and LS on PS yield a state that depends upon the order in which they are performed. This is contrary to the observation in a-Si:H [16]. Our inability to quench LS state by exposure to subbandgap light (IR) suggests that all metastable changes caused by the light exposures and thermal quenching might be related to the structural changes in PS or at PS/a-Si interface.

\section{EXPERIMENTAL}

Free standing PS flakes $(\sim 2 \times 5 \mathrm{~mm})$ were prepared by the electrochemical anodization of boron doped (100) crystalline silicon wafers having resistivity $\sim 1$ ohm- $\mathrm{cm}$. Interested readers are requested to see our previous report which says that our PS sample consists of crystallites of sizes $2-5 \mathrm{~nm}$ and bigger, measured by XRD and Atomic force microscope (AFM) [17]. For PL and ESR measurements, PS flakes were sealed in $10^{-1}$ torr Helium atmosphere in a quartz tube. The samples were annealed at $150{ }^{0} \mathrm{C}$ for 1 hour in vacuum to remove the effect of previous light exposures, if any, and slowly cooled (rate $\approx 0.5 \mathrm{~K} / \mathrm{min}$ ) to room temperature. This state of the sample after annealing and slow cooling shall be referred as "state A". For PL measurement, PS sample was excited by an Argon Ion laser (488 nm, 4 mW) and the emitted light was analyzed using a grating spectrometer and a CCD detector. 
ESR was measured using an X-band (Varian E-3) spectrometer at microwave power of $5 \mathrm{~mW}$. During electrical transport measurements about 70 micron thick flake of PS samples were mounted on Corning 7059 glass substrates by putting silver paint at the two ends of PS flakes, which also served as electrodes for current measurements. A bias of 40 volts was applied for the DC and PC measurements. PS showed a symmetric, sub ohmic I-V behavior upto this voltage. Light soaking (LS) was done in vacuum using heat filtered white light from a $250 \mathrm{~W}$ halogen lamp, kept at a distance of about $15 \mathrm{~cm}$ from the sample. After annealing (state A) precaution was taken to always keep the sample under dark conditions except for the light soaking experiments. For thermal quenching experiments, PS samples were annealed in vacuum at $150{ }^{0} \mathrm{C}(1 \mathrm{hr})$. Then the heater was switched off and chilled water was circulated through the copper block upon which the sample was mounted using silver paint without breaking the vacuum.

\section{RESULTS}

The ESR signal of our PS sample in annealed state (state A) is shown in figure 1. This signal is asymmetric and is similar to that reported by others [18]. It can be fitted (Fig.1, dotted line) to three signals; an isotropic signal with $\mathrm{g} \cong 2.0058 \pm 0.0006$ (Lorentzian, width $6.4 \pm 0.5 \mathrm{G}$ ) corresponding to the dangling bonds in a-Si and two anisotropic signals having $\mathrm{g} \perp=2.0081 \pm 0.0006$ (Gaussian, width $\sim 1.5 \pm 0.5 \mathrm{G}$ ) and $\mathrm{g}_{\mathrm{II}}$ $=2.0028 \pm 0.0006$ (Gaussian, width $\sim 1.8 \pm 0.5 \mathrm{G}$ ). The last two are attributed to the dangling bonds at the surface of PS [19]. This is in agreement with Watkins and Corbett [20] who suggested that $g_{\perp}$ varies from 2.0081 to 2.0110 and $g_{\text {II }}$ value varies from 2.0005 to 2.0026 for Si dangling bonds. Our observation is similar to Kondo et al. [21] who found an anisotropic ESR signal $\mathrm{g}_{\perp}=2.0096$ and $\mathrm{g}_{\mathrm{II}}=2.0031$ with a 
Gaussian broadening $3.2 \mathrm{G}$ using a powder pattern simulation for $\mathrm{P}_{\mathrm{b}}$ canters from $\mu$-Si (micro crystalline Si) samples. Our ESR data (Fig. 1) shows the signature of amorphous phase (about $6.4 \mathrm{G}, \mathrm{g} \sim 2.0058$ ) in our PS samples; because such broad ESR signal cannot arise from the nc-Si contribution. This is in agreement with Porkes and co workers who observed $8 \mathrm{G}$ broad ESR signal from porous silicon which signifies the presence of amorphous phase in PS [22]. This conclusion is in line with von-Bardeleben et al. [18]. Table 1 gives the summary of the different $g$ values with respective identity for the origin of the ESR signal available from the literature. Moreover, Kocka et al. as well as Lubianiker and Balberg have concluded the presence of amorphous phase from their respective electrical transport measurements on porous silicon $[23,24]$. A similar conclusion was also done by Ben-Chorin et al. $[25]$.

PL shows a single peak at about $800 \mathrm{~nm}$ with a full width at half maximum (FWHM) $\approx 130 \mathrm{~nm}$. It is assumed that PS consists of cylindrical crystallites of diameter $\mathrm{d}$ in which charges are confined in 2 dimensions. Initially, Canham and also Gosele and co workers have suggested that confinement of charge carriers in small crystallites is responsible for the enhancement of band gap in PS [26, 27]. A gaussian distribution of the wires of various diameters having a mean diameter $d_{o}$ is assumed to explain the broadening of PL spectrum [28]. The emission energy from a gaussian distribution of cylindrical crystallites is

$$
P\left(\Delta E_{u}\right)=\frac{N}{2 \Delta E_{u}}\left[\frac{c}{\Delta E_{u}}\right]^{\frac{3}{2}} \exp \left\{-\frac{1}{2}\left(\frac{d_{0}}{\sigma}\right)^{2}\left[\left(\frac{\Delta E_{0}}{\Delta E_{u}}\right)^{1 / 2}-1\right]^{2}\right\}
$$


where $\Delta E_{0}=\frac{c}{d_{0}^{2}}$-----------(2) is the up shift in energy associated with diameter $d_{0}$ and $c$ is $4.8 \mathrm{eV}-\mathrm{nm},{ }^{2}$ obtained from first principles calculations of Read and co workers [29]. The PL energy hv can be written as

$h v=\Delta E_{u}+\left(E_{g}-E_{b}\right)$

where $E_{g}=1.1 \mathrm{eV}$, the band gap of crystalline silicon and $\mathrm{E}_{\mathrm{b}}=0.15 \mathrm{eV}$, the exciton binding energy [28].

The energy up shift associated with a wire of diameter $5 \mathrm{~nm}$ can be calculated by using Eq. (2). The emission energy calculated from Eq. (3) will be $\sim 1.15 \mathrm{eV}$ for particle of diameter $5 \mathrm{~nm}$. Thus particles of diameter $5 \mathrm{~nm}$ and larger will give emission energy in infrared. For wires of diameters $\mathrm{d}_{01}=2.4 \mathrm{~nm}, \mathrm{~d}_{02}=3.4 \mathrm{~nm}$ and $\mathrm{d}_{03}$ $=4.6 \mathrm{~nm}$ with corresponding $\sigma_{1}=0.12 \mathrm{~nm}, \sigma_{2}=0.12 \mathrm{~nm}$ and $\sigma_{3}=0.12 \mathrm{~nm}$ respectively explain our experimental PL data (Fig. 2). Though the presence of a-Si phase in our PS sample is confirmed by ESR but major contribution to PL comes from the nanocrystalline part. In PS the intensity of PL increases as the temperature from $150 \mathrm{~K}$ to room temperature [30]. This is contrary to the results in a-Si:H [30, 31], which show that PL intensity decreases with increasing temperature from $4 \mathrm{~K}$ to room temperature. Thus a-Si:H may not be responsible for the emission of PL in visible from porous silicon. Brandt et al. compared the luminescence and vibrational properties of anodically oxidized PS and chemically synthesized siloxene $\left(\mathrm{Si}_{6} \mathrm{O}_{3} \mathrm{H}_{6}\right)$ [32]. Based on the quantitative agreement between these two types of materials they concluded that the origin of strong room temperature luminescence in porous silicon can be traced to siloxene derivates present in porous silicon. However, Tischler and Collins reviewed the arguments of siloxene model and reported that the FTIR (Fourier transform infrared spectroscopy) spectrum of the freshly prepared porous silicon, 
which had not been exposed to air, showed no detectable oxygen [33]. Based on this result they claimed that PL properties of porous silicon are inconsistent with the conclusion of Brandt and co workers [32]. Thus we believe that PL is caused by the quantum confinement of charge carriers in nanocrystalline Si core.

The effect of light exposures on DC, PC, PL and ESR of our PS samples is shown in figure 3. We see (Fig. 3) that DC, PC and PL increase for short light exposures but decrease for long exposures with a maximum at about $2700 \mathrm{sec}$. The new dark current persists for several hours even at room temperature. Exposure to infrared light $(<1.1 \mathrm{eV})$ is unable to restore state A. Annealing at $150{ }^{0} \mathrm{C}(1 \mathrm{hr})$, however, brings the sample to state A. The largest increase in DC is about a factor of 10 for an exposure of about $2700 \mathrm{sec}$. Further, for $2700 \mathrm{sec}$ exposure, PL intensity increases by about a factor of 3 and ESR decreases by $\approx 60 \%$ compared to the initial state A. On the other hand, we observe that for long exposures ESR increases with a decrease in DC, PC and PL. We have not noticed any change in PL peak position against light exposures.

The temperature dependence of PL during light soaking on another PS sample is shown in figure 4. For this, both light soaking and PL measurements were done at the same temperature. Curves (a), (b) and (c) in Fig.4 show the effect of light exposures on $\mathrm{PL}$ at $300 \mathrm{~K}, 325 \mathrm{~K}$ and at $355 \mathrm{~K}$ respectively. After each treatment vacuum annealing was done at $150{ }^{\circ} \mathrm{C}$ to erase the previous LS effect. At $300 \mathrm{~K}$ (curve (a) in Fig.4), PL intensity has a maximum at an exposure of $\sim 2700 \mathrm{sec}$. Curve (b) in Fig.4 shows that at 325K PL intensity increases with short LS with a maximum at an exposure of $1800 \mathrm{sec}$. At 355K (curve (c) of Fig.4) PL intensity does not change with short LS and then decreases for long exposures. We see that the maximum in PL occurs at lower exposure time as the temperature is increased. 
Fast cooling from $150{ }^{0} \mathrm{C}$ to room temperature $(400 \mathrm{~K} / \mathrm{min})$ brings PS to another metastable state (C), which has higher DC, PC and PL than the annealed state A (Table 2). This is also accompanied by a decrease in ESR signal. State A can be recovered by annealing at $150{ }^{\circ} \mathrm{C}(1 \mathrm{~h})$ and slow cooling, but not by IR exposure. In state $\mathrm{C}$ decrease in number of dangling bonds is responsible for the increase in PC and PL.

In order to see, if the light and thermal induced instabilities are caused mainly by the presence of a-Si in PS, we exposed our sample to cycles of LS and ammonia vapor as done by Tanielian 1982. Fig. 5 shows (cycle I) that the annealed state A (2.2 pA) goes to LS state after light soaking (30 min) with a higher DC (3.1 pA). A subsequent exposure to $\mathrm{NH}_{3}$ results in the final state $\mathrm{F}$, which has $\mathrm{DC}$ value $7.0 \mathrm{pA}$. Annealing at $150{ }^{\circ} \mathrm{C}$ brings the state $\mathrm{F}$ back to the state A. Now, if we reverse the order of $\mathrm{LS}$ and $\mathrm{NH}_{3}$ exposure, we obtain the state F' (cycle II, Fig.5) whose DC (4.8 $\mathrm{pA}$ ) is smaller than that of state $\mathrm{F}$ by about a factor of 1.5. Annealing restores the sample to state A, as before. This observation is at variance with the results of Tanielian 1982, who found that in a-Si:H, states $\mathrm{F}$ and F' are the same and the order of LS and ammonia exposure is unimportant.

\section{DISCUSSION}

Our ESR data (Fig. 1) shows the signature of amorphous phase (about $6.4 \mathrm{G}$, g 2.0058) in our PS samples; as such a broad ESR signal cannot arise from nc-Si contribution. This is in agreement with Prokes et al. [21] who observed 8 G broad peak from PS. They assigned this broad peak is from amorphous phase. We have also observed the presence of $\mathrm{g}_{\perp}=2.0081$ (width $\sim 1.5 \mathrm{G}$ ) and $\mathrm{g}_{\mathrm{II}}=2.0028 \pm 0.0006$ (width $\sim 1.8 \mathrm{G})$ in our porous silicon sample. A similar observation is made by Kondo et al. 
in case of $\mu$-Si samples [21]. Thus our ESR data is in line with von-Bardeleben et al. who observed that PS is a mixture of nanocrystalline and amorphous phases [18]. Therefore, porous silicon can be visualized as a mixture of nanocrystalline silicon with a-Si phase surrounded by hydrogen $[34,35]$. This is also in agreement with BenChorin et al., Kocka et al. and Lubianiker and Balberg [25, 23, 24]. Moreover, our ESR results completely ruled out the conclusion of Islam and Kumar [36].

Thus PS can be approximated as a mixture of a-Si and nc-Si phase. Light absorption occurs in nanocrystalline silicon core and photo-carriers diffuse to the surface where recombine. The dangling bonds act as nonradiative recombination centers. Thus, if the density of dangling bonds (ESR) decreases, PL should increase and vice versa. Indeed, we find (Fig.3) that ESR decreases for short exposures with an increase in PC and PL. Interestingly, ESR shows a minimum at about the same exposure duration $(2700 \mathrm{sec})$ at which DC, PC and PL show a maximum. For long exposures $(>3000 \mathrm{sec})$ ESR increases; accompanied by a decrease in PC and PL and therefore the usual Staebler-Wronski $[12,37]$ type mechanism might be operative. Furthermore, our short exposures results are in agreement with other authors $[6,7]$ and our long exposures results are in agreement with Matsumoto et al. [11] who also found that for long exposures dangling bond density (ESR) increases and PL decreases. A similar effect on DC for short as well as long exposures is observed in doping modulated and compensated a-Si:H [38, 39].

It is possible that the hydrogen present on the surface may provide the flexibility needed to rearrange the bond structure, though it is not known if hydrogen passivates whole PS layer or not. The rigid four-fold coordinated silicon cannot give such flexibility at room temperature [40]. Breaking of hydrogen from Si-H bond at room temperature is not possible, as it requires a large $(3 \mathrm{eV})$ energy. However, the 
energy needed for switching of Si-H bond is likely to be much smaller [13]. For short exposures, a hydrogen atom may move and bridge two nearby Si dangling bonds forming a $\mathrm{Si}-\mathrm{H}-\mathrm{Si}$, which may be strained, but give a decrease in number of dangling bonds (process-I). For longer exposures, hydrogen moves from one Si-H into a weak Si-Si bond creating one Si-H-Si metastable bond and one dangling bond (process-II) [40]. In general both these processes are likely to be operative during LS. Our results can be explained, if process-I is weaker but faster than process-II. Thus initially process-I dominates which decreases ESR. As LS is done for longer periods, processII takes over and we see a net increase in dangling bonds (ESR).

In an attempt to test the validity of the presence of the two processes mentioned above we measured the temperature dependence of LS on PL since it is unlikely that the two processes (I and II) have the same temperature dependence. We found (Fig. 4) that the maximum of the PL intensity shifts towards lower exposure times as the temperature increases. This is in consistent with the assumption of two processes mentioned above. Annealing at $150{ }^{\circ} \mathrm{C}$ brings the samples to the initial state. Moreover, curve ( c) of Fig. 4 shows that at $355 \mathrm{~K}$ light soaking causes only SW effect.

The thermal quenching effect shows an increase in DC, PC and PL and a decrease in ESR. Dark current increases by a factor of $\sim 50$ and photocurrent increases by a factor of about 1.2. A similar increase in dark current upon thermal quenching is observed in case of doped a-Si:H $[13,14,15]$. Interestingly, PL intensity increases by a factor of $\sim 2.6$ and ESR intensity decreases by about a factor of 1.2. Hydrogen movement in PS may be responsible for this behaviour, as suggested in the case of a-Si:H [13]. 
So far we have established a strong anti-correlation between DC, PC, PL and ESR. LS effects are not purely electronic but it seems that LS changes PS structurally. Short light exposures and thermal quenching cause a decrease of dangling bond density. On the other hand, long exposures result an increase of dangling bonds. Light soaking causes more than one type of defects. Two different types of processes are happening simultaneously while PS is soaked with light. One is PPC that saturates first and another one is similar to SW effect which is slower. However, we do not intend to say that the effect of LS on porous silicon sample can be measured by ESR completely. Moreover, it is not clear if surface of nc-si has an important role for the observed light induced effects or not.

There are various similarities between porous silicon and amorphous silicon. Light soaking for short exposures to porous silicon increases DC (PPC in a-Si:H) [6, $7,39,41]$ and long exposures decreases dark current (SW effect in a-Si:H). Both a$\mathrm{Si}: \mathrm{H}$ as well as PS recovers upon annealing above $150{ }^{\circ} \mathrm{C}$. An exposure to ammonia vapor increases dark current of porous silicon [8]. A similar increase in dark current in a-Si:H was observed by Tanielian [16]. A rapid thermal quenching increases DC in porous silicon (this work). Similar increase in dark current upon fast quenching was observed in doped a-Si:H by various authors $[13,14,15]$. Although the effect of LS and thermal quenching in PS are qualitatively similar to those reported for aSi:H, a closer look shows differences between the two. For example, the reduction in ESR for short exposures found in PS has not been reported so far in a-Si:H. Therefore, it appears that the presence of nanocrystalline $\mathrm{Si}$ also plays a role. Moreover, the observations of Fig. 5 is at variance with the results of Tanielian [16] who found that in a-Si:H, F and F' are the same and the order of LS and ammonia exposure is unimportant. In case of a-Si:H, it is believed that Staebler-Wronski 
effect is a bulk effect. Tanielian observed that the effect of light and exposure to ammonia vapour commute each other. He found that the order of LS and ammonia exposure is unimportant in a-Si:H. This is expected if light exposure creates bulk defect states in a-Si:H whereas ammonia vapour affects the surface only. Contrary to a-Si:H, we find that in PS the final state depends on the order in which LS and ammonia exposure are performed. Thus, our results cannot be understood in terms of the presence of a-Si in our sample. Further, in contrast with a-Si:H, it may imply that LS in PS affects the surface.

\section{CONCLUSIONS}

We find that PS sample is a mixture of nc-Si and amorphous phase. PS can be visualized as nc-Si particles embedded in amorphous silicon. Light absorption takes place at the nc-Si core and recombination takes place at the surface. Thus the surface dangling bonds play an important role for the metastabilities of PS samples. An increase in DC, PC and PL is observed when PS is exposed to light for a short duration $(<2700 \mathrm{~s})$. This is accompanied by a decrease in ESR signal. For exposures longer than $3000 \mathrm{~s}$ we get opposite effect. Fast cooling of PS to room temperature from $150{ }^{\circ} \mathrm{C}$ gives a state with higher $\mathrm{DC}, \mathrm{PC}$ and $\mathrm{PL}$, and is also accompanied by a decrease in ESR signal. Further, all metastable states can be annealed out at $150{ }^{0} \mathrm{C}$ (1h), but are stable against exposure to sub band gap (IR) light. This indicates that the observed changes cannot be explained by trapping of charges. Light soaking causes more than one type of defects in PS. Though these results are qualitatively similar to those observed in a-Si:H, a more careful look reveals the differences. For example, a decrease in ESR signal after LS or thermal quenching as we observed in PS has not been reported in a-Si:H. Similarly, we find that in PS the metastable state obtained by 
LS and ammonia exposures depends on the order in which they are performed. This is in contrary to the results in a-Si:H. This may mean that LS affects the surface in PS. This is in contrast to the a-Si:H where it is believed to be a bulk effect. We suggest that a local structural rearrangement of bonds involving movements of hydrogen at PS or at PS/a-Si interface is responsible for the LS and thermal quenching effect in PS.

\section{ACKNOWLEDGEMENTS}

We are grateful to Prof S. C. Agarwal for fruitful discussions. This work is supported by a grant from the Council of Scientific and Industrial Research, New Delhi, India.

\section{REFERENCES}

[1] O. Bisi, S. Ossicini and L. Pavesi, Surf. Sci. Reports, 38 (2000) p.1.

[2] L. Seals, J. L. Gole, L. A. Tse and P. J. Hesketh, J. Appl. Phys. 91 (2002) p.2519.

[3] V. Mulloni and L. Pavesi, Appl. Phys. Lett. 76 (2000) p.2523.

[4] P. Allcock and P. A. Snow, J. Appl. Phys. 90 (2001) p.5052.

[5] S. M. Prokes, W. E. Carlos, L. Seals and J. L Gole., Phys Rev. B 62 (2000) p. 1878 .

[6] W. H. Lee, H. Lee and C. Lee, J. Non-Crys. Solids 164-166 (1993) p.965.

[7] T. Frello, E. Veje and O. Leistiko, J. Appl. Phys. 79 (1996) p.1027.

[8] N. P. Mandal and S. C. Agarwal, J. Mater. Sci.: Mater. Electr. 14 (2003) p.797.

[9] Z. Y. Xu, M. Gal and M. Gross, Appl. Phys. Lett. 60 (1992) p.1375.

[10] M. A. Tischler, R. T. Collins, J. H. Stathis and J. C. Tsang, Appl. Phys. Lett. 60 (1992) p.639.

[11] T. Matsumoto, M. Kondo, S. V. Nair and Y. Masumoto, J. Non-Cryst Solids, 227-230 (1998) p.320. 
[12] D. L. Staebler and C. Wronski, Appl. Phys. Lett. 31 (1977) p.292.

[13] R. A. Street, J. Kakalios, C. C. Tsai and T. M. Hayes, Phys. Rev. B 35 (1987) p. 1316.

[14] Y. H. Song, B. S. Yoo, C. Lee and J. Jang, J. Non-Cryst. Solids 114 (1989) p.666.

[15] P. Agarwal and S. C. Agarwal, J. Appl. Phys. 81, (1997) p.3214.

[16] M. Tanielian, Phil. Mag. B 45 (1982) p.435.

[17] N. P. Mandal, S. Dey and S. C. Agarwal, Thin Solid Films, 451-452 (2004) p.75.

[18] H. J. von Bardeleben, D. Stievenard, A. Grosman, C. Ortega and J. Siekja, Phys. Rev. B 47 (1993) p.10899.

[19] J.C. Mao, Y.Q. Jia, J.S. Fu, E. Wu, B.R. Zhang, L.Z. Zhang and G.G. Qin, Appl. Phys. Lett. 62 (1993) p.1408.

[20] G. D. Watkins and J. W. Corbett, Phys. Rev. A, 134 (1964) p.1359.

[21] M Kondo, S Yamasaki and A. Matsuda, J. Non-Crys. Solids. 266-269 (2000) p.544.

[22] S. M. Porkes, W. E. Carlos and, V. M, Bermudez, Appl. Phys. Lett. 61 (1992) p. 1447.

[23] J. Kocka, I. Pelant and A. Fejfar, J. Non-Cryst. Solids. 198-200 (1996) p.857.

[24] Y. Lubianiker and I. Balberg, Phys. Rev. Lett. 78 (1997) p.2433.

[25] M. Ben-Chorin, F. Moller, F. Koch, W. Schirmacher and M. Eberhard, Phys. Rev. B 51 (1995) p.2199.

[26] L. T. Canham, Appl. Phys. Lett. 57 (1990) p.1046.

[27] V. Lehmann. and U. Gösele., Appl. Phys. Lett. 58 (1991) p.656.

[28] G. C. John and V. A. Singh, Phys. Rev. B 50 (1994) p.5329. 
[29] A.J. Read, R.J. Needs, K.J. Nash, L.T. Canham, P.D.J. Calcott and A.Qteish, Phys. Rev. Lett. 69(1993) p.1232

[30] L. Pavesi, Solid State Phenom. 44-46 (1995) p.261.

[31] F. Koch, V. Petrova-Koch and T. Muschik, J. Lumin. 57 (1993) p.271.

[32] M. S. Brandt, H. D. Fuchs, M. Stutzmann, J Weber and M Cardona, Solid State Commun. 81 (1992) p.307.

[33] M. A. Tischler and R. T. Collins, Solid State Commun. 84 (1992) p.819.

[34] Z. H. Lu, D. J. Lockwood and J. M. Baribeau, Nature 378 (1995) p.258.

[35] S. C. Agarwal, in Science and Technology of Nanostructred Materials, B. K. Rao, S. M. Bose, M. P. Das, and S. N. Sahu, eds., Nova, N.Y, 2001, p.101.

[36] Md. N. Islam and S. Kumar, Appl. Phys. Lett. 78 (2001) p.715.

[37] J. Kakalios, R. A. Street and W. B. Jackson, Phys. Rev. Lett. 59 (1987) p.1037.

[38] J. Kakalios and H. Fritzsche, Phys. Rev. Lett. 53 (1984) p.1602.

[39] S. C. Agarwal and S. Guha, Phys. Rev. B 32 (1985) p.8469.

[40] M. Stutzmann, W. B. Jackson and C. C. Tsai, Phys. Rev. B 32 (1985) p.23.

[41] N. P. Mandal, S. Dey and S. C. Agarwal, Mater. Res. Soc. Symp. Proc. Vol. 737 (2002) 
Table 1

Comparison of ESR $g$ values for Spin centers consisting of $\mathrm{P}_{b}$ centers, dangling bonds in nc-Si and dangling bonds in a-Si.

\begin{tabular}{|c|c|c|c|c|c|}
\hline Sample used & Identity & $\mathrm{g \perp}$ & $\mathrm{g}_{\mathrm{II}}$ & $\mathrm{g}$ & reference \\
\hline Crystalline Si & Dangling Bonds & $\begin{array}{l}2.0081- \\
2.0110\end{array}$ & $\begin{array}{l}2.0005- \\
2.0026\end{array}$ & - & $\begin{array}{l}\text { Watkins } \\
\text { and Corbett } \\
1964 \text {. }\end{array}$ \\
\hline Porous Si & Dangling Bonds & 2.0081 & 2.0026 & - & $\begin{array}{l}\text { Mao et al. } \\
1993 .\end{array}$ \\
\hline Porous $\mathrm{Si}$ & Dangling Bonds & - & - & 2.0055 & $\begin{array}{l}\text { Porkes et al. } \\
1992 .\end{array}$ \\
\hline$\mu-\mathrm{Si}$ & $\mathrm{P}_{\mathrm{b}}$ centre & 2.0078 & 2.0023 & - & $\begin{array}{l}\text { Kondo et al. } \\
2000 .\end{array}$ \\
\hline Porous Si & $\begin{array}{l}\mathrm{P}_{\mathrm{b}} \text { center and } \\
\text { dangling bond } \\
\text { signal from a-Si }\end{array}$ & 2.0091 & 2.0017 & 2.0055 & $\begin{array}{l}\text { von } \\
\text { Bardeleben, } \\
\text { et al. } 1993 \text {. }\end{array}$ \\
\hline Porous Si & $\begin{array}{l}\text { Dangling Bonds } \\
\text { from nc-Si and } \\
\text { a-Si }\end{array}$ & 2.0081 & 2.0028 & 2.0058 & this work \\
\hline
\end{tabular}


Table 2

DC, PC, PL and ESR measured at room temperature of a PS in the annealed state A, and after fast cooling (state C)

\begin{tabular}{|l|l|l|}
\hline & State A (Annealed) & State C (Quenched) \\
\hline Dark Current (DC) & $1.0 \times 10^{-12} \mathrm{~A}$ & $5.0 \times 10^{-11} \mathrm{~A}$ \\
\hline Photo Current (PC) & $4.6 \times 10^{-10} \mathrm{~A}$ & $5.5 \times 10^{-10} \mathrm{~A}$ \\
\hline Photoluminescence (PL) & $3.3 \pm 0.2$ a.u. & $8.8 \pm 0.2$ a.u. \\
\hline Dangling bonds (ESR) & $20.0 \pm 0.2$ a.u. & $16.6 \pm 0.2$ a.u. \\
\hline
\end{tabular}


Figure 1:

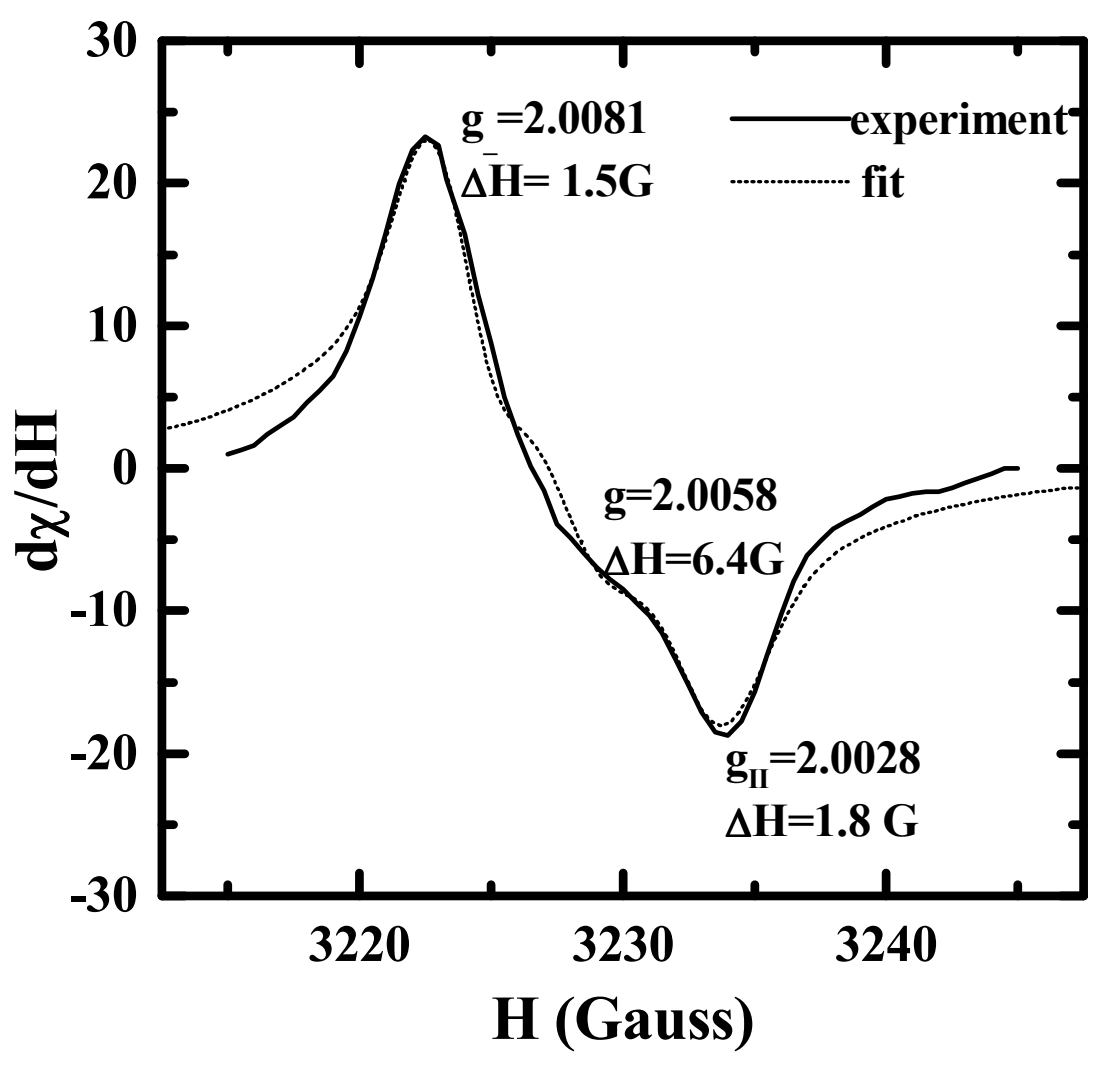

Fig.1: ESR signal in state A. Full line is the experiment and dotted line is the fit to three lines with g values and width shown. 
Figure 2:

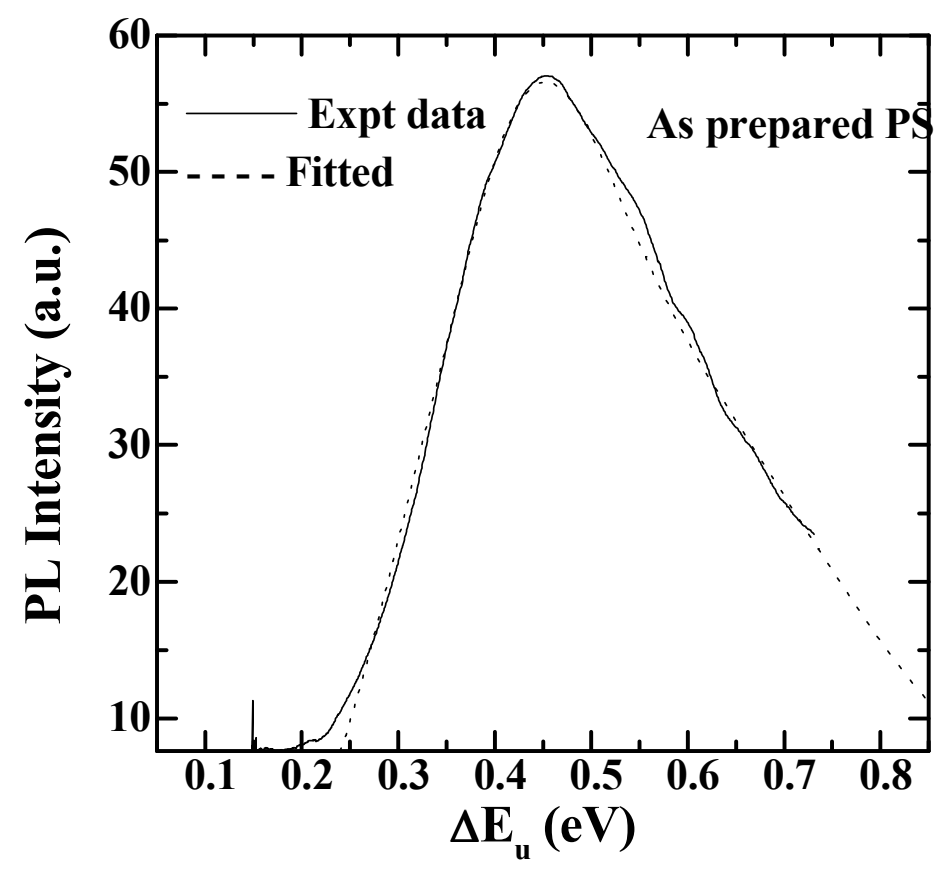

Fig. 2: PL from as prepared PS sample. The dotted line is the fitted data by JohnSingh model of quantum confinement. 
Figure 3:

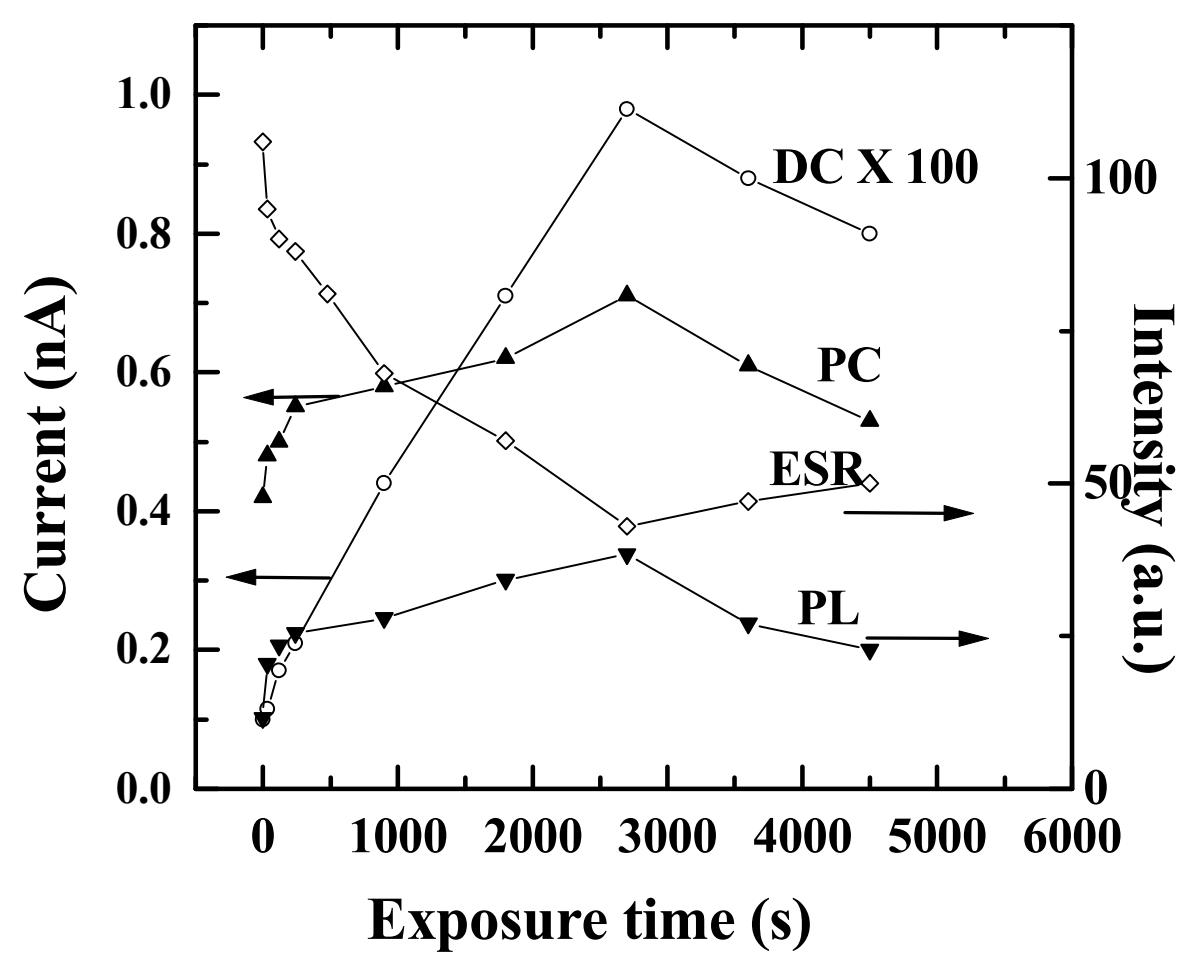

Fig. 3: Effect of light exposure on DC, PC, PL and ESR as a function of exposure time. The slope of all curves changes sign at the exposure time of $2700 \mathrm{~s}$. 
Figure 4:

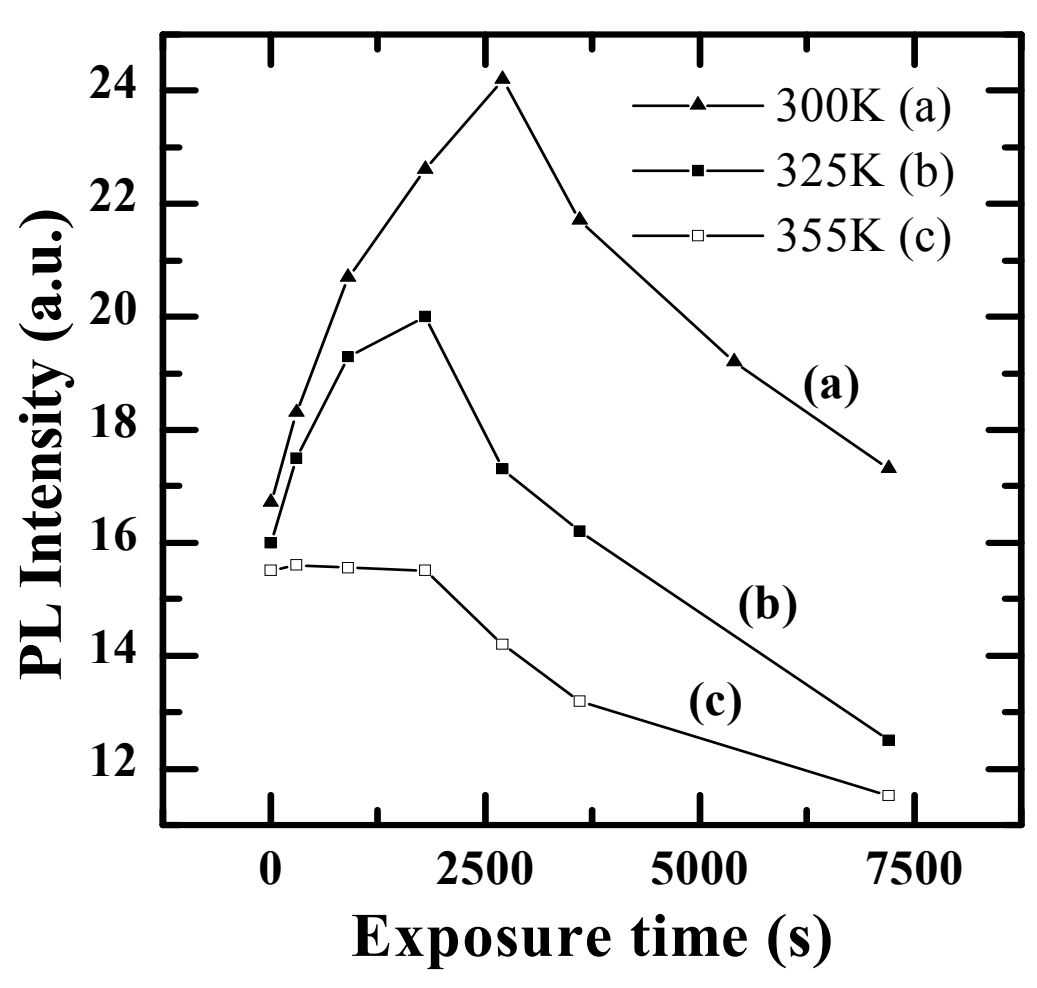

Fig. 4: The change in PL intensity with the duration of LS at (a) $300 \mathrm{~K}$, (b) $325 \mathrm{~K}$ and (c) $355 \mathrm{~K}$ respectively. 
Figure 5:

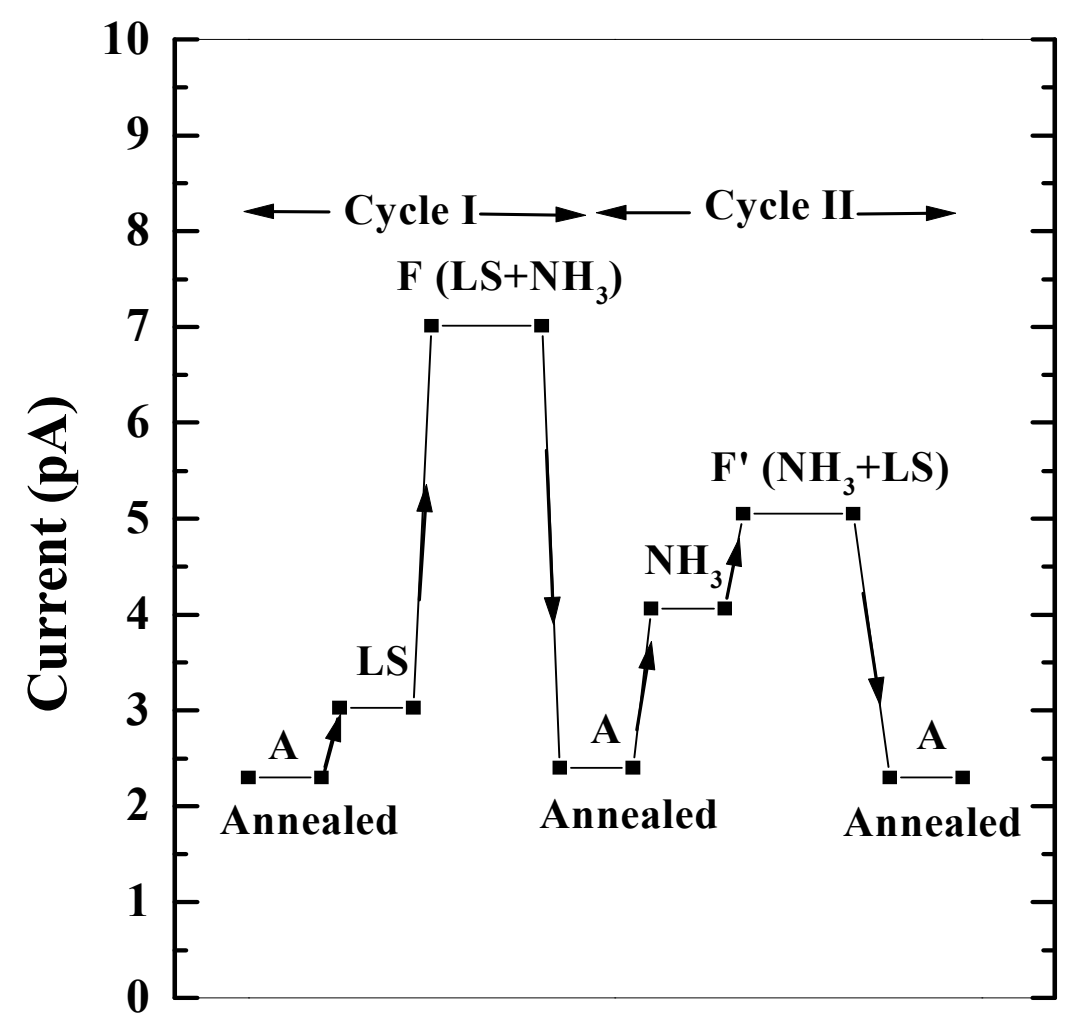

Fig. 5: Effect of 30 minutes LS followed by ammonia exposure (Cycle I) and effect of ammonia followed by 30 min LS (Cycle II) on DC of a free standing PS. Note that the state $\mathrm{F}$ obtained at the end of Cycle I is different than the state F' obtained after Cycle II. 


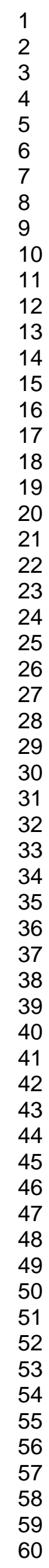

$215 \times 279 m m(600 \times 600$ DPI $)$

http://mc.manuscriptcentral.com/pm-pml 


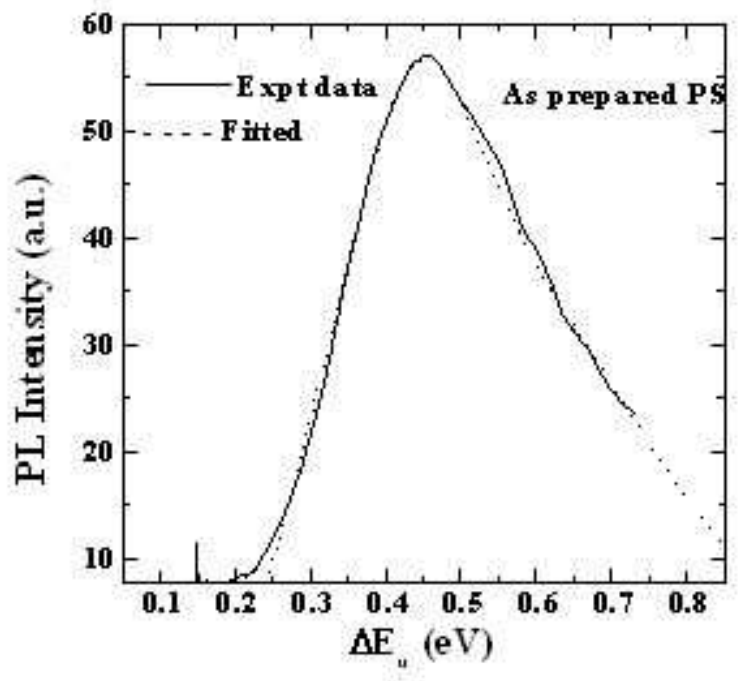

$81 \times 77 \mathrm{~mm}(96 \times 96 \mathrm{DPI})$

http://mc.manuscriptcentral.com/pm-pml 


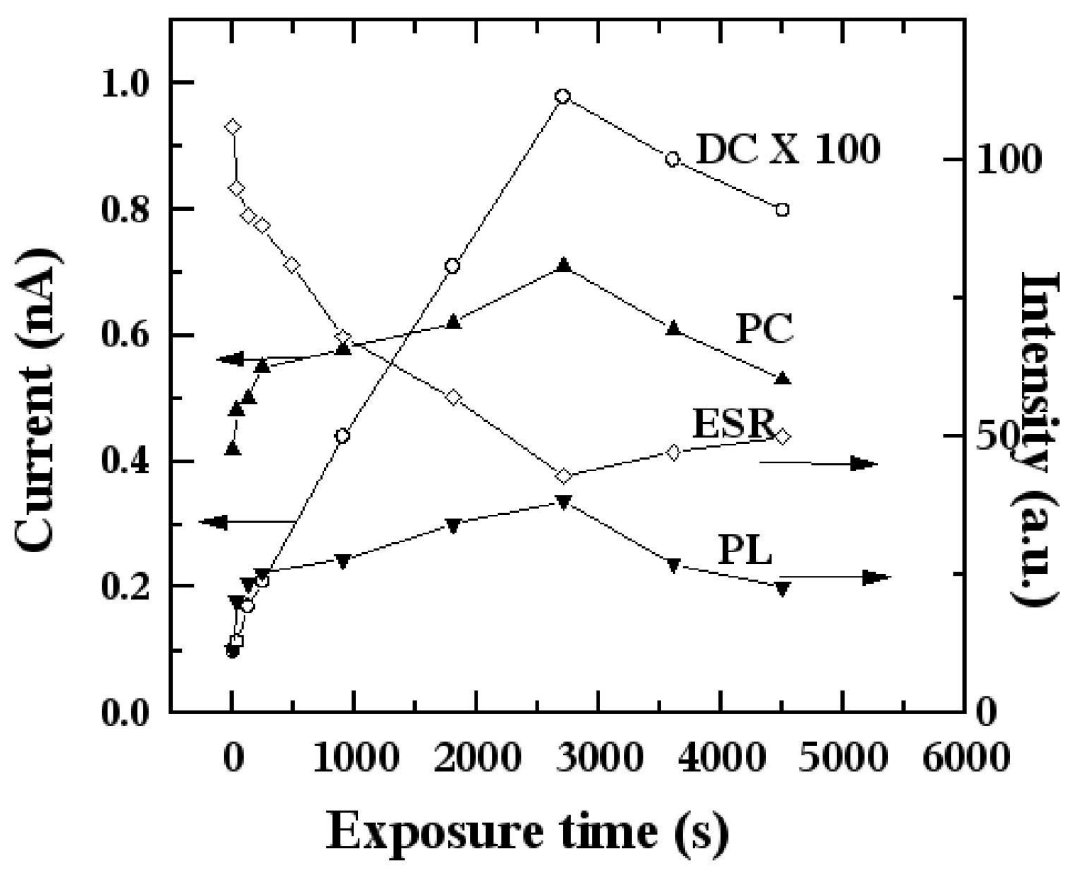

$215 \times 279 m m(600 \times 600$ DPI $)$ 


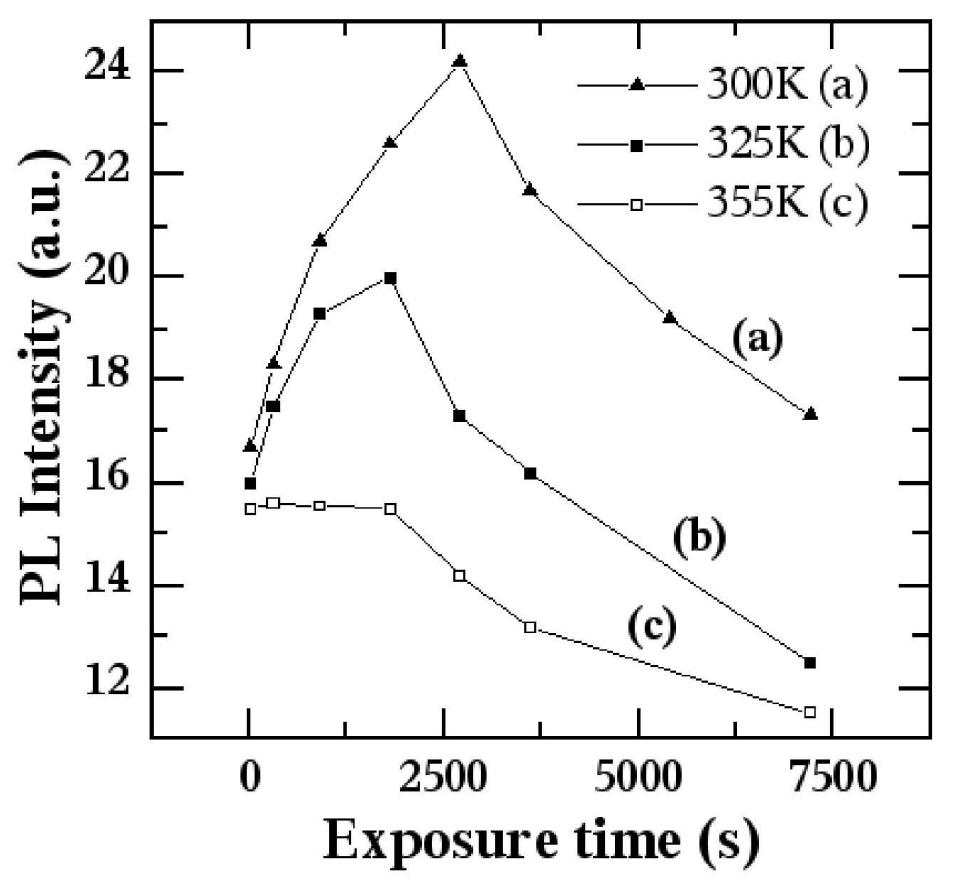

$215 \times 279 m m(600 \times 600$ DPI $)$ 


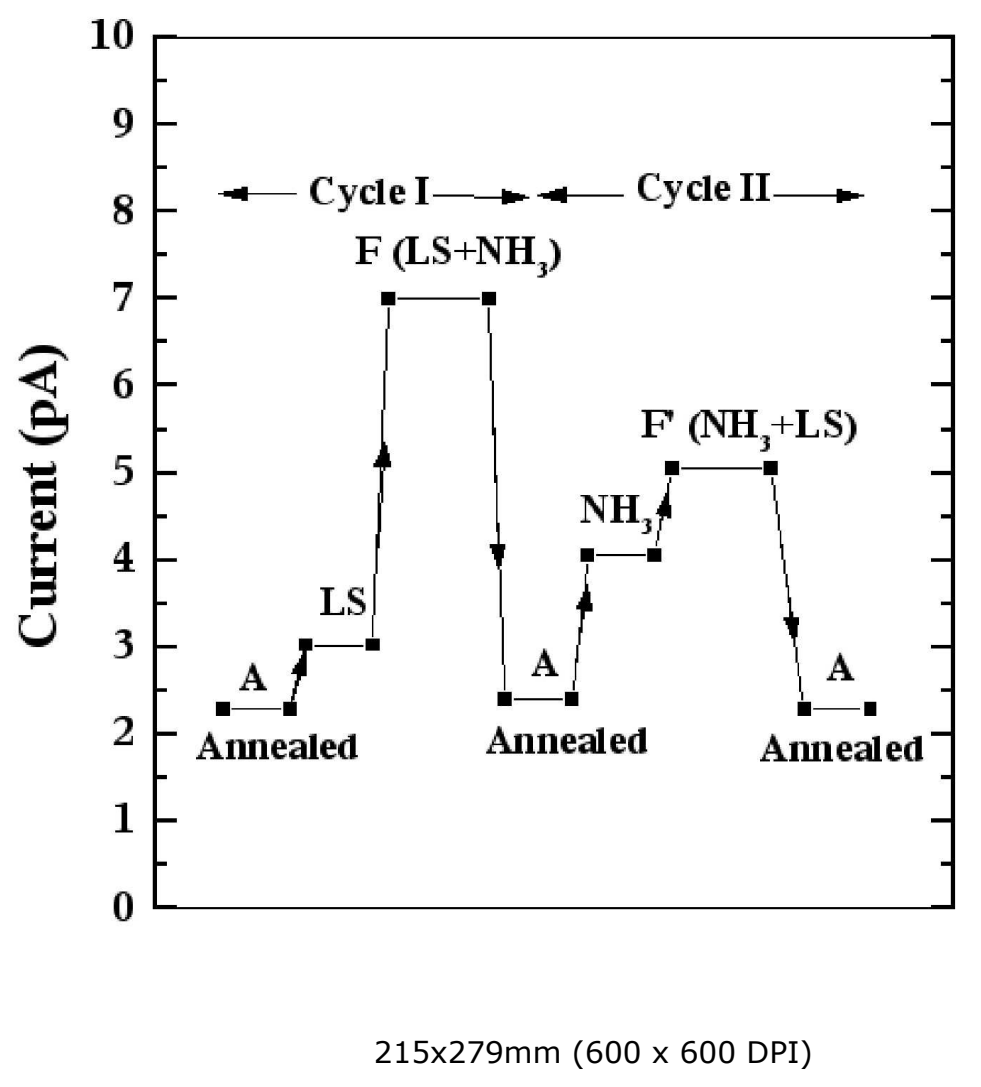

http://mc.manuscriptcentral.com/pm-pml 